tion enrolled thus far and to demonstrate the breadth and potential value of the data generated by the new CARRA Registry.

Methods We requested de-identified counts of several fields collected from the case report forms for subjects with SLE. Patients were eligible for enrollment in the new CARRA registry if they were diagnosed with SLE prior to the age of 18 and had either 1) a new diagnosis of SLE or 2) a flare of lupus nephritis within two years prior to the baseline visit. IRB approval was not required for this data request.

Results To date, 184 patients (pts) have been enrolled; 156 $(85 \%)$ are female. There are 46 black pts, 45 Hispanic pts, 47 white pts, 18 Asian pts and 16 pts were $>1$ race. Over half the pts have private health insurance $(n=95,52 \%)$ and 60 pts (33\%) have Medicaid. Autoantibody positivity was prevalent: 175 pts (95\%) were ANA positive, 109 (59\%) dsDNA positive, and 87 (47\%) anti-Smith positive. Positivity for anti-RNP, antiRo, anti-La, and APLs ranged from $15 \%$ to $51 \%$. At the baseline visit, the mean Systemic Lupus Erythematosus Disease Activity Index (SLEDAI, $\mathrm{n}=166$ ) score was $5.5 \pm 6.3$, median $=4$ (range 0-37; IQR 0.25-8). The mean Systemic Lupus International Collaborating Clinic Damage Index (SLICC DI, $n=150$ ) score was 0.4 , median $=0$ (range 0-7). Approximately one quarter of pts $(n=50)$ were being treated for lupus nephritis at the time of the baseline visit. Manifestations of SLE at the baseline visit were varied (table 1) but serologic disease, mucocutaneous disease and active nephritis were the most prevalent. Conclusions Nearly 200 SLE pts have been enrolled in the new CARRA Registry to date. This is a multi-racial cohort with moderate disease activity and varied disease manifestations. Further enrollment will continue to build a robust data source to study disease course and outcomes in a pediatric SLE inception cohort.

Acknowledgements The authors wish to acknowledge the Arthritis Foundation for ongoing financial support of CARRA and the CARRA Registry.

\section{CS-03 HYDROXYCHLOROQUINE IN LUPUS PREGNANCY: A META-ANALYSIS OF INDIVIDUAL PARTICIPANT DATA}

${ }^{1}$ Amanda M Eudy* ${ }^{2}$ Michelle Petri, ${ }^{3}$ Rebecca Fischer-Betz, ${ }^{4}$ Abeer Mokbel, ${ }^{5}$ Cecilia Nalli, ${ }^{5}$ Laura Andreoli, ${ }^{5}$ Angela Tincani, ${ }^{6}$ Yair Molad, ${ }^{7}$ Dafna Gladman, ${ }^{7}$ Murray Urowitz, 'Stephen Balevic, 'Megan EB Clowse. 'Duke University Medical Center; ${ }^{2} J o h n s$ Hopkins University School of Medicine; ${ }^{3}$ University Hospital Duesseldorf; ${ }^{4}$ Cairo University Hospital; ${ }^{5}$ Spedali Civili and University of Brescia; ${ }^{6}$ Tel Aviv University; ${ }^{7}$ University of Toronto

\subsection{6/lupus-2018-Ism.38}

Background Our current knowledge about how to treat lupus in pregnancy derives from small prospective or retrospective cohorts. The goal of this individual participant meta-analysis was to pool data from multiple prospective cohorts to answer the clinical question of whether hydroxychloroquine (HCQ) treatment affects pregnancy outcomes.

Methods The literature was searched for prospective cohorts of pregnancies among women with lupus. HCQ use was defined as use any time during pregnancy. Outcomes of interest included fetal loss, preterm birth, high disease, and preeclampsia. Data from each cohort were collected and analyzed individually. Pooled ORs were calculated by random-effect models in Review Manager. Due to multiple pregnancies per patient, one pregnancy was randomly selected per patient. Primary analysis included only women with first trimester visits (6 cohorts). Subgroup analyses were stratified by a history of nephritis, APS, and disease activity at first clinic visit.

Results The literature was searched for prospective cohorts of pregnancies among women with lupus. HCQ use was defined as use any time during pregnancy. Outcomes of interest included fetal loss, preterm birth, high disease, and preeclampsia. Data from each cohort were collected and analyzed individually. Pooled ORs were calculated by random-effect models in Review Manager. Due to multiple pregnancies per patient, one pregnancy was randomly selected per patient. Primary analysis included only women with first trimester visits (6 cohorts). Subgroup analyses were stratified by a history of nephritis, APS, and disease activity at first clinic visit.

Conclusions Our results suggest that among patients with lupus nephritis, HCQ use may decrease the risk of fetal loss. The heterogeneity of data collection suggests the need for a unified approach to identify larger cohorts of lupus pregnancies.

\section{CS-04 ASSOCIATED FACTORS OF LONG-TERM CARDIAC DYSFUNCTION IN A LONGITUDINAL COHORT OF NEONATAL LUPUS}

${ }^{1}$ Amit Saxena*, ${ }^{1}$ Peter M Izmirly, ${ }^{1}$ Rebecca Bomar, ${ }^{1}$ Shireen Golpanian, ${ }^{2}$ Deborah Friedman, ${ }^{1}$ jill P Buyon. 'NYU School of Medicine, New York, NY, USA; ${ }^{2}$ New York Medical College, Valhalla, NY, USA

\subsection{6/lupus-2018-Ism.39}

Background There are no longitudinal studies regarding the long term cardiac health of children with cardiac manifestations of neonatal lupus (NL). This study was performed to evaluate risk factors for morbidity and provide evidence-based guidance regarding the course of cardiac NL.

Methods Echocardiograms throughout life were evaluated in 240 individuals born with cardiac NL from the Research Registry for Neonatal Lupus: 142 were available from ages 01 years, 174 from ages 1-17 years, and 65>17 years. A composite adverse outcome defined as qualitatively decreased left ventricular (LV) function or concurrent use of cardiac

Abstract CS-03 Table 1 Pooled odds ratios for the association of hydroxychloroquine use and pregnancy outcomes

\begin{tabular}{|c|c|c|c|c|}
\hline & Fetal Loss & Preterm Birth & High Disease Activity & Preeclampsia \\
\hline & OR $(95 \% \mathrm{Cl})$ & OR $(95 \% \mathrm{Cl})$ & OR $(95 \% \mathrm{Cl})$ & OR $(95 \% \mathrm{Cl})$ \\
\hline Overall & $0.50(0.27-0.94)$ & $0.95(0.58-1.55)$ & $0.69(0.35-1.39)$ & $1.19(0.62-2.30)$ \\
\hline Lupus Nephritis History & $0.24(0.07-0.83)$ & $0.81(0.35-1.89)$ & $0.47(0.21-1.09)$ & $0.70(0.24-2.03)$ \\
\hline No Lupus Nephritis History & $0.70(0.33-1.46)$ & $1.04(0.57-1.89)$ & $0.98(0.45-2.17)$ & $1.36(0.58-3.16)$ \\
\hline APS & $0.39(0.10-1.47)$ & $0.82(0.23-2.96)$ & $1.30(0.16-10.48)$ & $0.55(0.12-2.45)$ \\
\hline No APS & $0.61(0.31-1.20)$ & $0.96(0.56-1.64)$ & $0.70(0.40-1.22)$ & $1.28(0.58-2.84)$ \\
\hline High Disease Activity at $1^{\text {st }}$ Visit & $0.61(0.13-2.89)$ & $1.53(0.42-5.62)$ & - & $0.93(0.12-7.14)$ \\
\hline No High Disease Activity at $1^{\text {st }}$ Visit & $0.46(0.21-1.02)$ & $0.81(0.45-1.44)$ & $0.73(0.29-1.87)$ & $1.07(0.50-2.31)$ \\
\hline
\end{tabular}


medications was assessed. Aortic dilation (root or ascending aorta $\mathrm{z}$-score $>2.0$ ) was also recorded. Analyses were performed to associate the composite adverse outcome and aortic dilation with maternal medications, pacing, and fetal disease status, including a severity score based on mortality risk factors such as lower fetal heart rate and extranodal disease.

Results The composite adverse outcome for cardiac dysfunction was identified in $21.1 \%$ of echos in children ages $0-1,13.2 \%$ ages $1-17 \%$ and $29.2 \%$ ages $>17$. In 89 children in which echos were available at ages $0-1$ and $1-17,6 / 16$ with dysfunction at ages $0-1$ were also affected at ages $1-17$, while 10 reverted to normal. Among those without dysfunction at age 0-1, 8/90 developed new worsening of cardiac function during age 1-17. In 35 cases with echos at ages $1-17$ and $>17,3 / 3$ cases with dysfunction at age $1-17$ were also affected at $>17$, and 2/32 developed new dysfunction in adulthood. Cardiac dysfunction was significantly associated with number of years paced at all ages $(\mathrm{p}<0.001,0.001$, $<0.001)$. A lower fetal ventricular heart rate at the first time of heart block detection was associated with cardiac dysfunction age $0-1$ and $>17$ ( $\mathrm{p}=0.048,0.005$ respectively) and lowest heart rate in utero associated with dysfunction at age $<1$ and 1-17 $(p<0.001,0.015)$. Fetal extranodal cardiac disease was associated with dysfunction in ages $1-17$ and $>17(p=0.026,0.023)$. Higher fetal severity score associated with postnatal dysfunction in ages $0-1$ and $1-17$ groups $(\mathrm{p}=0.013,0.001)$. Aortic dilation was present in $13.4 \%$ at ages $0-1 \%$ and $14.9 \%$ at ages $1-17$, but at $>17$, dilation only occurred in $9.2 \%$. There was no association of postnatal cardiac dysfunction or aortic dilation with maternal medication use, maternal rheumatic disease, fetal age at heart block detection or gestational age of birth.

Conclusions Cardiac dysfunction in the first year normalizes by later childhood in the majority of cases, possibly due to the short term effects of cardiac pacing or resolution of inflammation with the clearance of maternal autoantibodies. However, new onset dysfunction can occur after the first year of life. Aortic dilation can continue for longer periods, but may decrease in frequency with age. Nevertheless, cardiac dysfunction is present in roughly $30 \%$, and in adulthood there are associations with fetal extranodal disease and heart rate at detection. Patients who develop morbidity in utero may have subclinical damage or be more susceptible to future insults that manifest later in life, which can be exacerbated by prolonged pacing. Close monitoring and aggressive treatment of early extranodal disease in cardiac NL may have long term benefit in preventing subsequent morbidity.

\section{CS-05 CAN SYSTEMIC LUPUS ERYTHEMATOSUS (SLE) DISEASE ACTIVITY BE CONSISTENTLY SCORED AND INTERPRETED WITH SIMPLE, RAPID OUTCOME MEASURES?}

${ }^{1+}$ Anca D Askanase* ${ }^{2+}$ Aikaterini Thanou, ${ }^{2}$ Judith A James, ${ }^{2}$ Cristina Ariens, ${ }^{2}$ Teresa Aberle, ${ }^{2}$ Eliza Chakravarty, ${ }^{2} J o e$ Rawdon, ${ }^{2}$ Stan Kamp, ${ }^{3}$ Stavros Stavrakis, ${ }^{2}$ Joan T Merrill. ' Columbia University College of Physicians and Surgeons, New York, NY, USA; ${ }^{2}$ Oklahoma Medical Research Foundation, Oklahoma City, OK, USA; ${ }^{3}$ University of Oklahoma Health Sciences Center, Norman, OK, USA; + These authors share first authorship

10.1136/lupus-2018-Ism.40

Background Existing methods for grading lupus flares or improvement in disease activity require definition-based thresholds as increments of change. Visual analogue scales (VAS) allow rapid, continuous scaling of disease severity. We analyzed the performance of two scales, the SELENA SLEDAI
Physician's Global Assessment (SSPGA) and the Lupus Foundation of America-Rapid Evaluation of Activity in Lupus (LFAREAL $^{\mathrm{TM}}$ ) as measures of progress in SLE.

Methods Prospectively, we evaluated the agreement between collected disease activity (SLEDAI, BILAG-2004, CLASI, SSPGA and LFA-REAL ${ }^{\text {TM }}$ ) and response (SRI-4 and BICLA) measures in an ongoing clinical trial.

Results 50 patients (47 females, mean age $45 \pm 11.6$ years) were assessed at 528 consecutive visits (average $10.6 \pm 4.1$ visits per patient). Changes in disease activity compared to baseline were examined in 478 visit pairs. SSPGA and LFAREAL $^{\mathrm{TM}}$ correlated with each other $(r=0.936)$, and with SLEDAI and BILAG [SSPGA: $\quad r=0.742 \quad$ (SLEDAI), $\quad r=0.776$ (BILAG); LFA-REAL ${ }^{\mathrm{TM}}$ : $\mathrm{r}=0.778$ (SLEDAI), $\mathrm{r}=0.813$ (BILAG); all $\mathrm{p}<0.0001]$. Changes $(\Delta)$ in SSPGA and LFA-REAL ${ }^{\mathrm{TM}}$ compared to screening correlated with each other $(r=0.857)$ and with changes in SLEDAI and BILAG [ $\triangle$ SSPGA: $r=0.678$ $(\triangle \mathrm{SLEDAI}), \quad \mathrm{r}=0.624 \quad(\triangle \mathrm{BILAG}) ; \quad \Delta \mathrm{LFA}^{-R_{E A L}{ }^{\mathrm{TM}}: \quad \mathrm{r}=0.686}$ $(\triangle$ SLEDAI), and 0.700 ( $\triangle$ BILAG); all $p<0.0001]$. Changes in SSPGA and LFA-REAL ${ }^{\text {TM }}$ strongly correlated with SRI-4 and BICLA by ROC analysis $(p<0.0001$ for both, see figure 1 below). Additionally, LFA-REAL ${ }^{\mathrm{TM}}$ correlated to individual BILAG organ scores [musculoskeletal: $r=0.842$, mucocutaneous: $r=0.826$ ( $p<0.0001$ for both)].

Conclusions SSPGA and LFA-REAL ${ }^{\text {TM }}$ are reliable surrogates of common SLE trial endpoints and could be used as continuous or dichotomous response measures. Additionally, LFA-REAL ${ }^{\mathrm{TM}}$ can provide individualized scoring at the symptom or organ level.

Acknowledgements We thank the patients for their participation in the study. Clarification of Abatacept Effects in SLE with
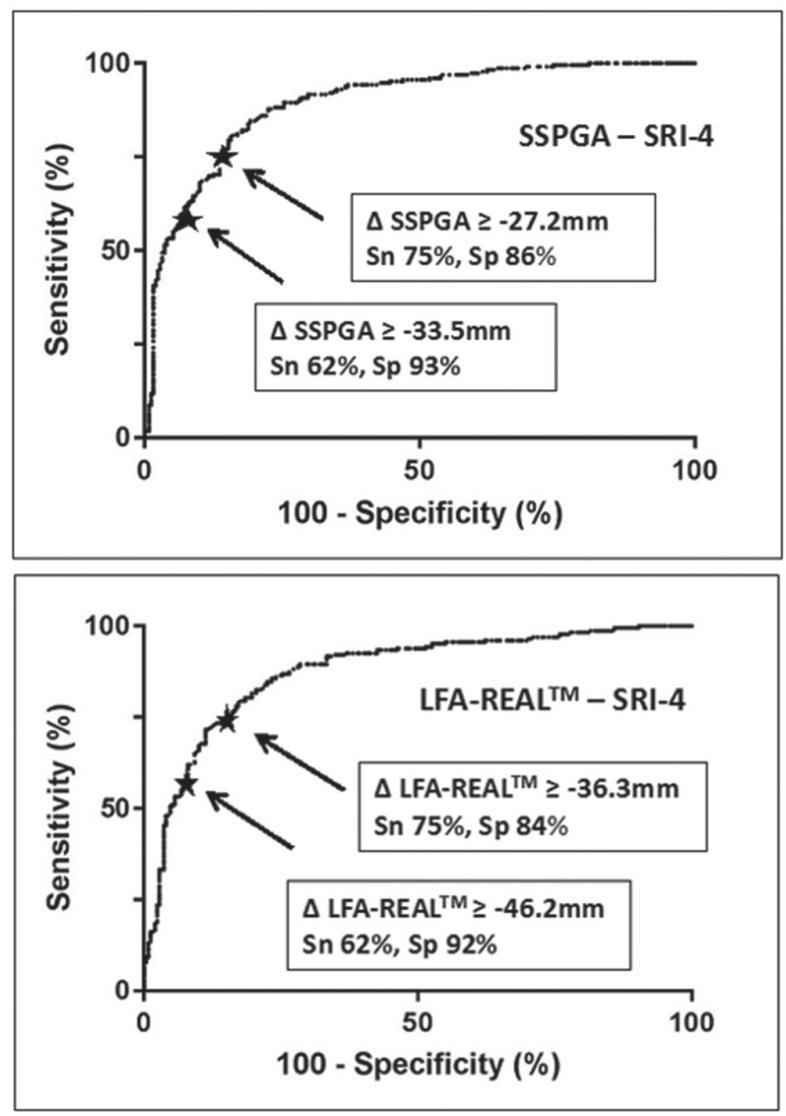

Abstract CS-05 Figure 1 ROC analysis for SSPGA-SRI-4 and LFAREAL $^{\text {TM }}-$ SRI-4 